\title{
Evaluation of Informed Consent for Withholding and Withdrawal of Life Support in Korean Intensive Care Units
}

\author{
Jin Ha Park, M.D., Shin Ok Koh, M.D., Ph.D. , Jin Sun Cho, M.D., Ph.D., and Sungwon Na, M.D., Ph.D. \\ Department of Anesthesiology and Pain Medicine, and Anesthesia and Pain Research Institute, Yonsei University College of Medicine, and *Department of Anesthesiology and Pain \\ Medicine, Chung-Ang University College of Medicine, Seoul, Korea
}

Background: The goal of this study was to analyze the process and characteristics of withholding or withdrawal of life support (WLS) in Korean intensive care units (ICUs).

Methods: This was a single-centered retrospective analysis of patients who died in the ICUs of a tertiary hospital in Korea from January to December 2012. WLS informed consents and clinical data were analyzed.

Results: Of 285 deaths during the study period, informed consents for WLS were obtained from 228 patients ( $80.0 \%)$. All WLS decisions were made by family members after the patient's loss of decision-making capacity. Decisions were made most frequently by the patient's son (50.6\%). Patients in the WLS group were older than those in the non-WLS group, and older age was associated with the WLS decision. Thirty-seven patients (16.2\%) died within one hour of WLS approval, and 182 patients (79.8\%) died on the day of WLS approval. The most frequently withheld life support modality was chest compression (100\%), followed by defibrillation (95.9\%) and pacemaker insertion (63.3\%).

Conclusions: Aggressive and invasive life support measures were those most frequently withheld or withdrawn by decision-makers in Korean ICUs. The most common proxy was the son, rather than the spouse.

Key Words: informed consent; intensive care units; life support care; withholding treatment.

\section{Introduction}

End-of-life care is a major issue in medical ethics due to the advancement of life support technology.[1] Intensive care may prolong the dying process in patients who are unresponsive to treatment already provided.[2] The concept of withholding or withdrawing of life support (WLS) was introduced to limit the suffering of critically ill patients. Decisions regarding WLS are difficult and affected by several factors including not only disease severity, but also ethics, religion, culture, and legal background.[3] In Western countries, advance directives play an important role in WLS for dying patients and honor patient autonomy. However, advance directives in Korea have not been supported by law and culture under Confucianism, which re-

Received on October 9, 2014 Revised on February 24, 2015 Accepted on February 24, 2015

Correspondence to: Sungwon Na, Department of Anesthesiology and Pain Medicine, and Anesthesia and Pain Research Institute, Yonsei University College of Medicine, 50 Yonsei-ro, Seodaemun-gu, Seoul 120-752, Korea

Tel: +82-2-2228-2420, Fax: +82-2-312-7185

E-mail: NSWKSJ@yuhs.ac

*No potential conflict of interest relevant to this article was reported. quires offspring to do their best to treat parents in the name of filial piety, making decisions difficult for physicians and family members with terminally ill patients.[4,5] WLS in the intensive care unit was typically decided without any official documentation in Korea before doctors were punished by the supreme court for aiding and abetting murder for withdrawing life support from dying patients.[6] Little information exists

(c) This is an Open Access article distributed under the terms of the Creative Commons Attribution Non-Commercial License (http://creativecommons.org/ licenses/by-nc/3.0/) which permits unrestricted non-commercial use, distribution, and reproduction in any medium, provided the original work is properly cited. 
about end-of-life practices in Korean ICUs. Most ICU patients do not have the capacity to express their own opinions about WLS and informed consent has usually been given by family members. We have used informed consent in clinical practice for terminally ill patients in our hospital.

We sought to investigate the proportion and characteristics of informed consent for WLS of patients who died in the ICUs, as documented by the family, and to compare the patient characteristics of the WLS group with those of the non-WLS group.

\section{Materials and Methods}

\section{1) Patient population}

This study was conducted after approval from the institutional review board (IRB number, 4-2012-0858) of Severance Hospital and informed consent was exempted due to the retrospective nature of data analysis. This study was registered at www.ClinicalTrials.gov (ref. number: NCT02020473). We reviewed all consecutive patients who died in the surgical and medical intensive care units (ICUs) at Severance Hospital, Korea from January to December 2012. Severance Hospital is a tertiary teaching hospital and the ICUs in this study are composed of four units with a 54-bed and open system capable of the critical care services necessary for all ICU patients. WLS was typically recommended for terminally ill patients by the ICU team or primary physicians, when further treatment was considered futile. Modalities of treatments withhold or withdraw included chest compression, defibrillation, pacemaker insertion, use of vasopressors or increase dose of vasopressors, intubation, mechanical ventilation, use of antiarrhythmic drugs, transfusion, nutrition, blood sampling, use of antibiotics and dialysis. Patients diagnosed with brain death were excluded because they were admitted to the ICU solely for organ donation. Patients who died in other cardiac, cardiothoracic, stroke and neurosurgical intensive care units were not included in our study. Patients under 18 years were also excluded.

\section{2) Collected clinical and WLS information}

Information was obtained from electronic medical records. We collected demographics, clinical data of patients, and data regarding informed consent for WLS. Clinical data included underlying diseases, primary cause of ICU admission and WLS decision, Acute Physiology and Chronic Health Evaluation II score (APACHE II score) and Sequential Organ Failure Assessment score (SOFA score) at the time of ICU admission and WLS decision, length of stay in the ICU and hospital, time interval from ICU admission to WLS decision, time interval from WLS decision to death, operation before ICU admission, cardiopulmonary resuscitation (CPR) before ICU admission and WLS decision, clinical status of patients at the time of ICU admission and WLS decision, and clinical status of patients without WLS before death. Data regarding WLS included characteristics of the family members who signed WLS consent and type of the life support modalities withheld or withdrawn. Subgroup analysis was performed in patients with malignancy for more detailed information.

\section{3) Statistical analysis}

All statistical analyses were performed using SPSS 18 (SPSS Inc., Chicago, IL, USA). All continuous variables including age, hospital length of stay and time interval from ICU admission to WLS decision were analyzed with the Student $\mathrm{t}$ test or Mann-Whitney $\mathrm{U}$ test, as determined by the equality of variances and distribution. Chi-square and Fisher's exact tests were calculated for all categorical vari-

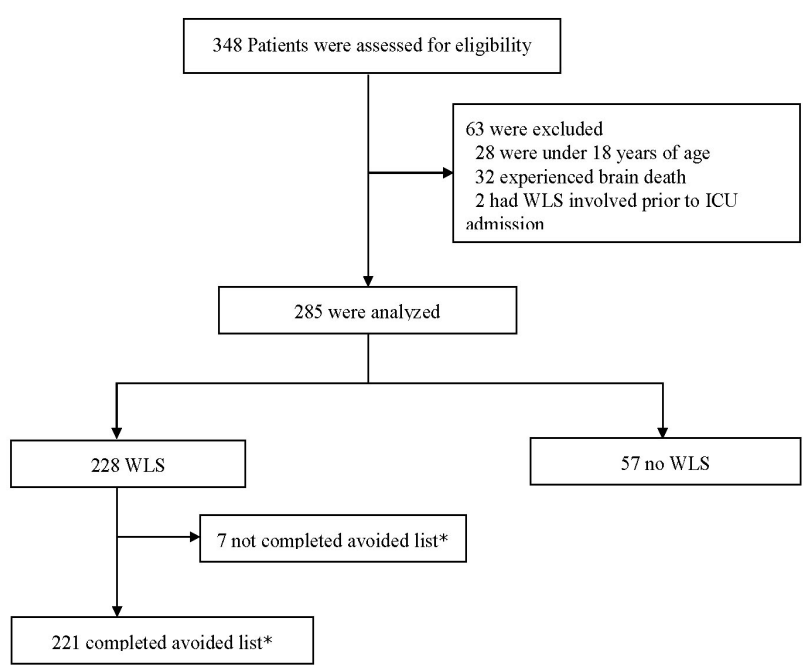

Fig. 1. Patient flow chart. *Avoided list; list of withheld/withdrawn actions on the informed consent form for WLS. ICU: Intensive Care Unit; WLS: withholding and withdrawal of life support. 
ables, as appropriate. Univariate analysis was subsequently performed to identify potential factors associated with WLS decision. Variables with $\mathrm{p}<0.20$ on univariate modeling and with clinical importance were included in multivariate analysis. Entry of factors into the model was verified by multicollinearity testing. A separate logistic regression model was also generated to identify predictors of WLS decision.

\section{Results}

Over the 1-year period of the study, 2,685 patients were admitted to the medical and surgical ICUs. We reviewed
348 consecutive patients who died in the ICUs. Of these patients, we excluded the following patients: 28 patients under 18 years of age, 32 patients with brain death, two cases involving WLS prior to ICU admission, and 1 patient who died of an unknown cause. Finally, we enrolled 285 patients and assessed the demographic and medical data of these patients. Informed consent for WLS was obtained from 228 patients $(80.0 \%)$. Seven patients in WLS group did not complete the list for which life support modalities would be withheld or withdrawn (Fig. 1).

Table 1 shows the baseline characteristics of the patients in the WLS and the non-WLS group. Patients in the WLS group were older than patients in the non-WLS group ( $p$

Table 1. Baseline characteristics of the patients with/without withholding or withdrawal of life support care

\begin{tabular}{|c|c|c|c|}
\hline Parameter & WLS $(n=228)$ & non-WLS $(n=57)$ & $\mathrm{p}$-value \\
\hline Age (year) & $64.35 \pm 14.04$ & $58.77 \pm 18.48$ & 0.037 \\
\hline Male, n (\%) & $155(68.0)$ & $32(56.1)$ & 0.092 \\
\hline \multicolumn{4}{|l|}{ Length of Stay (day) } \\
\hline Hospital & 19 [9-39] & $17[6-37]$ & 0.360 \\
\hline ICU & 9 [3-18] & $6[1-14]$ & 0.110 \\
\hline \multicolumn{4}{|l|}{ Past medical history, n (\%) } \\
\hline Malignancy & $136(59.6)$ & $34(59.6)$ & 0.999 \\
\hline Hypertension & $106(46.5)$ & $27(47.4)$ & 0.905 \\
\hline Diabetes & $55(24.1)$ & $13(22.8)$ & 0.835 \\
\hline Heart disease & $49(21.5)$ & $9(15.8)$ & 0.339 \\
\hline Lung disease & $42(18.4)$ & $9(15.8)$ & 0.643 \\
\hline Kidney disease & $42(18.4)$ & $11(19.3)$ & 0.879 \\
\hline Liver disease & $30(13.2)$ & $5(8.8)$ & 0.367 \\
\hline Neurologic disorder & $19(8.3)$ & $10(17.5)$ & 0.040 \\
\hline Gastrointestinal disease & $8(3.5)$ & $2(3.5)$ & 0.999 \\
\hline CPR at general ward, $n(\%)$ & $35(15.4)$ & $11(19.3)$ & 0.469 \\
\hline OP before ICU admission, n (\%) & $46(20.2)$ & $14(24.6)$ & 0.468 \\
\hline \multicolumn{4}{|c|}{ Primary causes for ICU admission, n (\%) } \\
\hline Respiratory failure & $134(58.8)$ & $26(45.6)$ & 0.073 \\
\hline Septic shock & $51(22.4)$ & $18(31.6)$ & 0.147 \\
\hline Cardiac arrest & $28(12.3)$ & $9(15.8)$ & 0.481 \\
\hline Hypovolemic shock & $20(8.8)$ & $7(12.3)$ & 0.418 \\
\hline Renal failure & $12(5.3)$ & $6(10.5)$ & 0.218 \\
\hline Neurologic disorder & $10(4.4)$ & $0(0.0)$ & 0.220 \\
\hline Hepatic failure & $7(3.1)$ & $2(3.5)$ & 0.999 \\
\hline Heart failure & $5(2.2)$ & $1(1.8)$ & 0.999 \\
\hline
\end{tabular}

Data might be duplicated.

CPR: cardiopulmonary resuscitation; ICU: Intensive Care Unit; OP: operation; WLS: withholding and withdrawal of life support. 
$<0.05)$. Gender and length of stay in the hospital and ICU were not significantly different between the groups. About $60 \%$ of the patients had malignancy, and $45 \%$ of patients had a history of hypertension. There was no significant difference in past history between the two groups except for lower incidence of neurologic disorder $(p=0.040)$ in the WLS group. Cardiopulmonary resuscitation (CPR) and operation history before admission to the ICU were not significantly different between the two groups. The most common primary causes for ICU admission were respiratory failure and septic shock in the two groups and there was no significant difference between the two groups (Table 1).

Table 2 demonstrates the clinical status of the two groups of patients. Mental state was clear in $15.4 \%$ of patients in the WLS group and $8.8 \%$ in the non-WLS group $(\mathrm{p}=0.201)$. About $82.9 \%$ of patients in WLS group and $86.0 \%$ of patients in the non-WLS group had already been mechanically ventilated with intubation $(p=0.576)$. Sedatives were given for $76.8 \%$ of patients in the WLS group and for $75.4 \%$ in the non-WLS group $(p=0.834)$. Analgesics were given for $75.4 \%$ of patients in both groups $(p>0.999)$. Vasopressors were used in $71.1 \%$ of patients in the WLS group and $75.4 \%$ in the non-WLS groups $(\mathrm{p}=0.501)$. Dialysis was performed in $35.5 \%$ of patients in the WLS group, and $56.1 \%$ of patients in the non-WLS group at admission to the ICU (p = 0.004). At the time of WLS decision, the clinical status of patients had changed; the percentage of patients with alert mental status decreased to $2.6 \%$, use of vasopressors increased from $71.1 \%$ to $90.8 \%$, the proportion of patients mechanically supported with intubation increased to $96 \%$, the proportion of analgesics and sedatives given increased from $75-77 \%$ to $83 \%$ and dialysis increased from $35.5 \%$ to $63.6 \%$. The APACHE II score on admission to the ICU was 26 [20-31] and 28 [18-33] in patients in the WLS group and non-WLS group, respectively $(\mathrm{p}=0.189)$. At the time of WLS decision, the APACHE II score increased to 31.[28-

Table 2. Clinical status of patients with/without withholding or withdrawal of life support care

\begin{tabular}{|c|c|c|c|}
\hline Parameter & WLS $(n=228)$ & non-WLS $(n=57)$ & p-value \\
\hline \multicolumn{4}{|c|}{ At the time of admission to ICU, $\mathrm{n}(\%)$} \\
\hline Intubated & $189(82.9)$ & $49(86.0)$ & 0.576 \\
\hline Mechanical ventilation & $189(82.9)$ & $49(86.0)$ & 0.576 \\
\hline Use of sedatives & $175(76.8)$ & $43(75.4)$ & 0.834 \\
\hline Use of analgesics & $172(75.4)$ & $43(75.4)$ & 0.999 \\
\hline Use of vasopressors & $162(71.1)$ & $43(75.4)$ & 0.510 \\
\hline Dialysis & $81(35.5)$ & $32(56.1)$ & 0.004 \\
\hline Alert mental status & $35(15.4)$ & $5(8.8)$ & 0.201 \\
\hline \multicolumn{4}{|l|}{ At the time of WLS, n (\%) } \\
\hline Intubated & $220(96.5)$ & & \\
\hline Mechanical ventilation & $220(96.5)$ & & \\
\hline Use of vasopressors & $201(90.8)$ & & \\
\hline Use of sedatives & $189(82.9)$ & & \\
\hline Use of analgesics & $188(82.5)$ & & \\
\hline Dialysis & $145(63.6)$ & & \\
\hline Alert mental status & $6(2.6)$ & & \\
\hline APACHE II score, ICU admission & 26 [20-31] & 28 [18-33] & 0.189 \\
\hline APACHE II score, WLS decision & 31 [28-35] & & \\
\hline SOFA score, ICU admission & $13[9-16]$ & $13[11-16]$ & 0.315 \\
\hline SOFA score, WLS decision & 18 [16-20] & & \\
\hline
\end{tabular}

Data might be duplicated.

APACHE II score: Acute Physiology and Chronic Health Evaluation II score; ICU: Intensive Care Unit; SOFA score: Sequential Organ Failure score; WLS: withholding and withdrawal of life support. 
Table 3. Clinical data of patients in WLS group

\begin{tabular}{|c|c|}
\hline Parameter & WLS $(n=228)$ \\
\hline CPR before WLS, n (\%) & $14(6.1)$ \\
\hline \multicolumn{2}{|l|}{ Direct causes for WLS, n (\%) } \\
\hline Refractory shock & $116(50.9)$ \\
\hline Respiratory failure & $108(47.4)$ \\
\hline Multiorgan failure & $47(20.6)$ \\
\hline Hepatic failure & $17(7.5)$ \\
\hline Brain damage & $16(7.0)$ \\
\hline Heart failure & $13(5.7)$ \\
\hline Renal failure & $11(4.8)$ \\
\hline Median time from ICU to WLS (hour) [range] & 156 [38-327] \\
\hline Median time from WLS to death (hour) [range] & 9 [3-20] \\
\hline \multirow{2}{*}{\multicolumn{2}{|c|}{$\begin{array}{l}\text { Data might be duplicated. } \\
\text { CPR: cardiopulmonary resuscitation; ICU: Intensive Care Unit; WLS: withholdin } \\
\text { and withdrawal of life support. } \\
\text { Table 4. The lists of withheld/withdrawn actions on the informe } \\
\text { consent form for WLS }\end{array}$}} \\
\hline & \\
\hline $\begin{array}{l}\text { Avoided lists in the informed consents for } \\
\text { WLS }(n=221)\end{array}$ & $\mathrm{n}(\%)$ \\
\hline Chest compression & $221(100)$ \\
\hline Defibrillation & $212(95.9)$ \\
\hline Pacemaker & $140(63.3)$ \\
\hline Increase dose of vasopressors & $107(48.4)$ \\
\hline Antiarrhythmic drugs & $75(33.9)$ \\
\hline Dialysis & $71(32.1)$ \\
\hline Transfusion & $45(20.4)$ \\
\hline Use of vasopressors & $39(17.6)$ \\
\hline Blood sampling & $28(12.7)$ \\
\hline Nutrition & $25(11.3)$ \\
\hline Antibiotics & $16(7.2)$ \\
\hline Mechanical ventilation & $10(4.5)$ \\
\hline Intubation & $9(4.1)$ \\
\hline
\end{tabular}

Data might be duplicated.

WLS: withholding and withdrawal of life support.

Table 5. Main family member who made the WLS decision

\begin{tabular}{lc}
\hline Legal representatives $(\mathbf{n}=\mathbf{2 2 8})$ & $\mathbf{n}(\%)$ \\
\hline Son & $121(53.1)$ \\
Spouse & $45(19.7)$ \\
Daughter & $36(15.8)$ \\
Mother & $4(1.8)$ \\
Father & $3(1.3)$ \\
etc. & $19(8.3)$ \\
\hline
\end{tabular}

WLS: withholding and withdrawal of life support.
35] The SOFA score on admission to the ICU was 13 [9$16]$ in patients in the WLS group and 13 [11-16] in the nonWLS group $(p=0.315)$. The SOFA score was also increased 18 [16-20] at the time of WLS decision.

Table 3 shows clinical data of patients in the WLS group. In the WLS group, the median time from ICU admission to making the decision for WLS was 156 [38-327] hours and median time from WLS decision to death was 9 [3-20] hours. Thirty-seven patients (16.2\%) died within 1 hour of WLS approval, and 182 patients (79.8\%) died on the day of WLS approval. The most frequent cause leading to WLS decision was refractory shock accompanied by respiratory failure $(50.9 \%, 47.4 \%$, respectively (Table 3$)$.

Table 4 shows the lists of withheld/withdrawn actions on the informed consent form for WLS. No patient had signed informed consent to withdraw life support modalities which had already been applied. Among patients with a list of actions to be withheld on the informed consent form for WLS $(n=221)$, the most frequently withheld life support modality was chest compression $(n=221,100 \%)$, followed by defibrillation $(\mathrm{n}=212,95.9 \%)$, pacemaker insertion $(\mathrm{n}=$ 140, $63.3 \%)$, and increased dose of vasopressor $(\mathrm{n}=107$, 48.4\%). Endotracheal intubation was withheld in 9 patients (4.1\%), mechanical ventilation in 10 patients $(4.5 \%)$, and nutrition in 25 patients (11.3\%) (Table 4).

Table 5 shows the family members who made the WLS decision. The main family members who signed informed consent for WLS were sons $(\mathrm{n}=121,50.6 \%)$ and spouses ( $\mathrm{n}$ $=45,19.7 \%)$, followed by daughters $(n=36,15.8 \%)$. Fortyone patients in the non-WLS group died after CPR (71.9\%). Among these patients, 11 patients (19.3\%) agreed to stop resuscitation in the end, but they did not sign informed consents for WLS.

Table 6 shows the multivariate logistic regression analysis of WLS on patient variables. When age was included in the multivariate logistic regression model, it was associated with WLS decision (odds ratio 1.023, CI 1.004-1.043, $\mathrm{p}=$ 0.004). History of neurologic disease also had a significant association (odds ratio 0.322, CI 0.135-0.768, $\mathrm{p}=0.011$ ), whereas other variables were eliminated from the model (Table 6).

Subgroup analysis in patients with malignancy was done as follows: 17 patients in the WLS group were alert at the time of ICU admission, whereas none of patients in the non- 
Table 6. Logistic regression analysis of WLS on patient variables

\begin{tabular}{|c|c|c|c|c|}
\hline & \multicolumn{2}{|c|}{ Univariate analysis } & \multicolumn{2}{|c|}{ Multivariate analysis } \\
\hline & 0dds ratio (95\% Cl) & $p$-value & Odds ratio (95\% Cl) & $\mathrm{p}$-value \\
\hline Age & $1.023(1.005-1.042)$ & 0.014 & $1.028(1.009-1.048)$ & 0.004 \\
\hline LOS_ICU & $1.021(0.997-1.046)$ & 0.086 & & \\
\hline History of neurologic disease & $0.427(0.187-0.978)$ & 0.044 & $0.322(0.135-0.768)$ & 0.011 \\
\hline \multicolumn{5}{|l|}{ Direct causes for ICU admission } \\
\hline Respiratory failure & $1.700(0.948-3.048)$ & 0.075 & & \\
\hline Septic shock & 0.624 (0.329-1.183) & 0.149 & $0.546(0.282-1.057)$ & 0.073 \\
\hline
\end{tabular}

ICU: Intensive Care Unit; LOS: length of stay; WLS: withholding and withdrawal of life support.

WLS group was alert $(\mathrm{p}=0.026)$. The median time from ICU admission to WLS decision was 139 [29-274] hours, though 156 [38-327] hours in whole population of the WLS group (see supplementary Table 1-5).

\section{Discussion}

Our study investigated the pattern of end-of-life decisions and practices in Korean ICUs. In the present study, a large proportion of the patients and decision makers declined invasive or aggressive procedures including chest compression, defibrillation and pacemaker insertion. Sons were the preferred decision makers in families, even when spouses were present. The rate of WLS was $80 \%$, as high as that of Western ICUs. The median time from WLS to patient death was 9 hours.

Invasive and aggressive management and procedures were often withheld by decision makers. On the other hand, they were more apt to continue nutrition, antibiotics and blood sampling. Among life support therapies, intubation, mechanical ventilation, use of antibiotics and nutrition were rarely chosen to be withheld. This might be influenced by the attitude of Korean people towards oxygen and nutrition, as well as use of antibiotics, which are considered ordinary medical practices instead of extraordinary life support measures.[7,8] Although frequently withheld modalities were chest compression, defibrillation and pacemaker insertion, similar to the results of a previous study,[9] discrepancies between perceptions by physicians and the general public still exist. Physicians are more likely to consider the withholding of nutrition, antibiotics and antiarrhythmics than the general public, as shown in our study. This discrepancy may result from the opinion of physicians, many of whom think that quality of life is most important and aggressive treatments are not preferable in end-of-life care,[10] and patients' misconceptions about end-of life care.[11] International differences were also found, in that intubation, application of ventilator and dialysis, use of vasopressors and antibiotics, and dialysis were withheld less often than reported in previous studies.[12-14]

In general, making patients' their own medical decisions is more common than delegating the right to a family member. Although Koreans want to make medical decisions by themselves and prefer to delegate decisions to their spouses when they are unable,[3] offspring, especially sons, were the main family members who decided WLS in this study. In our study, in $53.1 \%$ of cases, informed consent for WLS was signed by sons, $15.8 \%$ by daughters and $19.7 \%$ by spouses. The reason for sons being the main decision makers might be as follows: parents often become dependent financially and emotionally on their adult children after their retirement, even though they remain capable of decision making. Thus, many discussions, including on medical issues, are decided by patients' offspring, suppressing the expression of opinions by spouses. Preference for sons has a long tradition in Korea, and is still quite common. This is evidenced by a report that the sex ratio of newborns of multiparous women increased from 128 in the second birth to 417 in the fourth birth in cases where the first baby was a girl.[15]

Informed consent for WLS was obtained for $80.0 \%$ of patients who died in the ICUs. This result was similar to studies in Western ICUs, $[16,17]$ while ICUs in Asia and the Middle East where there is a culture of paternalism have reported much lower rates of WLS: 45.7-58.8 \%.[13,14] Additionally, most decisions regarding WLS were made 
just before death, similar to in European countries.[17,18] Therefore, the medical decision pattern in Korean ICUs is basically comparable to that of the Western world, while still showing traditional differences such as a preference for sons as decision makers. The relatively higher incidence of WLS in our study might be related to the short median time interval from WLS decision to death, as most patients became more critically ill prior to the WLS decision. This is similar to a study by Sprung et al[17] which showed a 14.7hour mean time from WLS decision to death and a $76 \%$ incidence of WLS, whereas Buckley et al.[13] described a relatively longer mean time to death and a lower incidence of WLS. Previous studies have shown an increase in WLS from $51 \%$ to $90 \%,[16]$ and these trends are expected to continue as life support technology progresses.

All WLS decisions were made by family members in the absence of decision-making capacity in patients. Korea is an Asian country and is influenced by Confucianism, which emphasizes filial piety, and discussions about dying and the death of parents are considered to be taboo. In these circumstances, it could be inferred that life support therapy continued until treatment became medically futile, with the purpose of preparing the remaining family for separation from patients, [8] and for avoiding being blamed for neglecting their parents.[19]

Old age was identified as the biggest factor that influenced WLS decision, similar to previous studies.[12,13,17,20-22] Gender did not have any significance in these decisions, whereas Nakagawa, et al. described a tendency to receive early DNR orders from female patients.[23] This could be explained by the fact that Koreans are not familiar with expressing their wishes about end of life treatment regardless of gender.

Patients with neurologic disease were not prone to WLS in our study, whereas neurologic disease influenced WLS in a previous study.[24] This might be influenced by the end-oflife care policy in our hospital, which supports patients who have already made decisions regarding WLS in the general ward instead of admitting them to the ICU. This may be supported by the fact that there were only 10 patients who were admitted to the ICU because of neurologic disease. The most common reason for ICU admission was respiratory failure in both the WLS group and non-WLS group in our data. This is a similar to the results of a previous study.
[25] The major causes for WLS did not differ from previous studies and were refractory shock and respiratory failure.

In our study, no patient decided to withdraw life support. At ICU admission and WLS decision time, most patients were supported with a mechanical ventilator, and sedation and analgesic was given for the mechanically supported patients. Most patients were also given vasopressors at ICU admission and at WLS decision time. In Korea, withdrawing life support is not acceptable, not only because it is considered different from withholding life support for that family members abandon the patients, but also it is still not supported by law.[4,26,27] In these circumstances, advance directives can be introduced,[28] which request withholding a particular intervention in a terminal status, withholding resuscitation, appointment of a proxy decision maker, and a living will. Although advance directives are not supported by law in Korea, more discussions about advance directives are needed in order to not prolong futile treatment in patients with terminal status, and previous studies have displayed positive attitudes toward advance directives.[5,29]

The strength of the present study is that all data collected were obtained from officially documented discussions with the family. Thus, this study could help to establish end-oflife care guidelines for critically ill patients who have already lost decision-making ability regarding end of life care at admission to the ICU. A strategic approach to limiting life support is necessary, as demonstrated in the present study, in which most patients who died in the ICU did not have alert mental status and had already been treated with life support modalities including mechanical ventilation or vasopressors at admission.

There were several limitations to the present study. Firstly, this study was designed retrospectively, and there might be unknown or unmeasured confounders which affected the WLS decision, such as the religion of patients and families, who initiated the discussion of WLS, and how many people were involved in the WLS decision. We could only determine who signed informed consent for WLS. Secondly, it was hard to determine the direct reason leading to the WLS decision, such as futility of treatment, disclosure of suffering of patients or other economic reason. Thirdly, this study was performed in medico-surgical intensive care units with 54 beds, and other intensive care units for cardiologic patients with 24 beds, cardiothoracic 15-bed units for surgical 
patients, neurosurgical 30-bed units and 8-bed stroke units were not included in this study. Our results showed a lower incidence of primary admission for neurologic disease in patients in the WLS group and thus the results may not be able to be generalized to other patients in other medical settings, even in the Korean population.

The decision on whether or not life support therapy should be initiated in critically ill patients involves balancing the potential for ensuring best practice to the patients against meaningless treatment. Aggressive and invasive life support measures were those most frequently withheld by decision makers in Korean ICUs. The most common proxy was the son, rather than the spouse. The present study provides an opportunity to establish end of life care guidelines for critically ill patients in Korea who have lost the decisionmaking capacity for WLS, and for whom advance directives are not supported by law.

\section{Acknowledgements}

We would like to thank our statistical assistants Dong Wook Kim and Jung Hwa Hong for their help with data analysis.

\section{References}

1) Jensen HI, Ammentorp J, Johannessen H, Ørding H: Challenges in end-of-life decisions in the intensive care unit: an ethical perspective. J Bioeth Inq 2013; 10: 93 101.

2) Moon JY, Shin YS: The end-of-life care in the intensive care unit. Korean J Crit Care Med 2013; 28: 163-72.

3) Ivo K, Younsuck K, Ho YY, Sang-Yeon S, Seog HD, Hyunah B, et al: A survey of the perspectives of patients who are seriously ill regarding end-of-life decisions in some medical institutions of Korea, China and Japan. J Med Ethics 2012; 38: 310-6.

4) Ryu HG, Choi JE, Lee S, Koh J, Bae JM, Heo DS: Survey of controversial issues of end-of-life treatment decisions in Korea: similarities and discrepancies between healthcare professionals and the general public. Crit Care 2013; 17: R221.
5) Jo KH, An GJ: Factors affecting shared decision making at end of life in Korean adults. Holist Nurs Pract 2013; 27: 329-35.

6) Supreme Court Decision 2002Do995 Decided June 24, 2004.

7) Aita K, Takahashi M, Miyata H, Kai I, Finucane TE: Physicians' attitudes about artificial feeding in older patients with severe cognitive impairment in Japan: a qualitative study. BMC Geriatr 2007; 7: 22.

8) Kim SH, Son MS, Koh SO, Lee IH: Intensive care provider's perceptions of medically futile treatment: Focus group interviews. Korean J Med Ethics 2012; 15: 37084.

9) Kim S, Kang H, Koh Y, Koh S: Attitudes and practices of critical care physicians in end-of-life decisions in Korean intensive care units. Korean J Med Ethics 2009; 12: $12-28$.

10) Sprung CL, Carmel S, Sjokvist P, Baras M, Cohen SL, Maia P, et al: Attitudes of European physicians, nurses, patients, and families regarding end-of-life decisions: the ETHICATT study. Intensive Care Med 2007; 33: 104-10

11) Morita T, Miyashita M, Shibagaki M, Hirai K, Ashiya T, Ishihara $\mathrm{T}$, et al: Knowledge and beliefs about end-oflife care and the effects of specialized palliative care: a population-based survey in Japan. J Pain Symptom Manage 2006; 31: 306-16.

12) Esteban A, Gordo F, Solsona JF, Alia I, Caballero J, Bouza $\mathrm{C}$, et al: Withdrawing and withholding life support in the intensive care unit: a Spanish prospective multi-centre observational study. Intensive Care Med 2001; 27: 1744-9.

13) Buckley TA, Joynt GM, Tan PY, Cheng CA, Yap FH: Limitation of life support: frequency and practice in a Hong Kong intensive care unit. Crit Care Med 2004; 32: 415-20.

14) Yazigi A, Riachi M, Dabbar G: Withholding and withdrawal of life-sustaining treatment in a Lebanese intensive care unit: a prospective observational study. Intensive Care Med 2005; 31: 562-7.

15) Lee JK, Cho KI, Park CH, Cho SJ: Last 10 year's statistics of newborn baby's sex ratio in multigravide at Taegu Catholic Medical Center. Korean J Obstet Gynecol 1993; 36: 3565-75. 
16) Prendergast TJ, Luce JM: Increasing incidence of withholding and withdrawal of life support from the critically ill. Am J Respir Crit Care Med 1997; 155: 15-20.

17) Sprung CL, Cohen SL, Sjokvist P, Baras M, Bulow HH, Hovilehto S, et al: End-of-life practices in European intensive care units: the Ethicus Study. JAMA 2003; 290: 790-7.

18) Lee K, Jang HJ, Hong SB, Lim CM, Koh Y: Do-notresuscitate order in patients, who were deceased in a medical intensive care unit of an university hospital in Korea. Korean J Crit Care Med 2008; 23: 84-9.

19) Li LB: Clinical review: Ethics and end-of-life care for critically ill patients in China. Crit Care 2013; 17: 244.

20) Ferrand E, Robert R, Ingrand P, Lemaire F; French LATAREA Group: Withholding and withdrawal of life support in intensive-care units in France: a prospective survey. French LATAREA Group. Lancet 2001; 357: 9-14.

21) de Camargo Cancela M, Comber H, Sharp L: Age remains the major predictor of curative treatment nonreceipt for localised prostate cancer: a population-based study. Br J Cancer 2013; 109: 272-9.

22) Hamel MB, Teno JM, Goldman L, Lynn J, Davis RB, Galanos AN, et al: Patient age and decisions to withhold life-sustaining treatments from seriously ill, hospitalized adults. SUPPORT Investigators. Study to Understand Prognoses and Preferences for Outcomes and
Risks of Treatment. Ann Intern Med 1999; 130: 116-25.

23) Nakagawa K, Vento MA, Seto TB, Koenig MA, Asai SM, Chang CW, et al: Sex differences in the use of early do-not-resuscitate orders after intracerebral hemorrhage. Stroke 2013; 44: 3229-31.

24) Kranidiotis G, Gerovasili V, Tasoulis A, Tripodaki E, Vasileiadis I, Magira E, et al: End-of-life decisions in Greek intensive care units: a multicenter cohort study. Crit Care 2010; 14: R228.

25) Azoulay E, Metnitz B, Sprung CL, Timsit JF, Lemaire F, Bauer P, et al: End-of-life practices in 282 intensive care units: data from the SAPS 3 database. Intensive Care Med 2009; 35: 623-30.

26) Heo DS: Life-sustaining medical treatment for terminal patients in Korea. J Korean Med Sci 2013; 28: 1-3.

27) National Evidence-based Healthcare Collaborating Agency. Social consensus for withdrawal of futile lifesustaining treatments. Seoul, Korea, 2009.

28) Hartog CS, Peschel I, Schwarzkopf D, Curtis JR, Westermann I, Kabisch B, et al: Are written advance directives helpful to guide end-of-life therapy in the intensive care unit? A retrospective matched-cohort study. J Crit Care 2014; 29: 128-33.

29) Sun DS, Chun YJ, Lee JH, Gil SH, Shim BY, Lee OK, et al: Recognition of advance directives by advanced cancer patients and medical doctors in hospice care ward. Korean J Hosp Palliat Care 2009; 12: 20-6. 